\title{
Towards a Goal-Driven Approach for Business Process Improvement Using Process-Oriented Data Warehouse
}

\author{
Khurram Shahzad and Constantinos Giannoulis \\ Department of Software and Computer Systems, Royal Institute of Technology (KTH), \\ Stockholm, Sweden \\ mks@dsv.su.se \\ Department of Computer and Systems Science, Stockholm University (SU), \\ Stockholm, Sweden \\ constantinos@dsv.su.se
}

\begin{abstract}
An emerging approach for business process analysis is to use business intelligence practices by employing data warehouse and decisionmaking techniques. However, little work has been done on developing core methods and tools to guide process analysis and improvement. Our research addresses this issue by introducing a goal-driven approach for business process improvement using process warehouse. In this paper, we present our three step method and its evaluation through an empirical study. The results showed that the impact of applying our method for process improvement has been perceived positively.
\end{abstract}

Keywords: Process Improvement, Business Process Intelligence, Businessorientation of Process Warehouse, Goal-driven Process Improvement.

\section{Introduction}

Analysis and improvement of business processes, a core phase of business process management (BPM) lifecycle, has been receiving increasing attention among many enterprises. Facilitating sustainable process improvement requires an understanding of processes as well as approaches for continuously analyzing and improving them.

An emerging approach for business process analysis utilizes business intelligence practices, which attempts to boost the analytical capabilities of BPM systems by employing process-oriented data warehouse $[1,2]$, called process warehouse (PW) [3]. PW captures the data about executed business processes, such as actors, activities performed by actors, resources used, execution time and thereby can be used as an adequate basis for analysis and optimization of those processes [2, 3, 4].

Due to a typically large size of PW and lack of approaches for their efficient use, users commonly retrieve irrelevant data, miss vital information, and as a consequence, fail in the process analysis task [5]. Muehlen et al. [6] has argued that if the cognitive effort to extract and interpret the information from a process warehouse is high, the extracted information may be ignored and thus it doesn't bring any value for decision makers. It is because, users are accustomed to the business perspective in process analysis [7] and thereby far from capabilities to directly utilize the data 
contained in warehouses for process analysis and improvement. Therefore, the question arises, how can process warehouse be used for analysis and improvement of business processes? An additional problem is that despite the PW concept exists for some years, only a limited number of implementations are reported (e.g. HP's business process cockpit [8]). A possible reason for this is the lack of methods that can guide business process improvement using process warehouse [5, 9].

In order to address the outlined problems, a starting point may be the consideration of the needs for business process analysts and decision makers. They are accustomed to the business perspective in the process analysis [10] and earlier studies [7, 11] contend they prefer to analyze business performance in accordance to goals. Therefore, our approach to address the discussed issues is based on a business orientation in the design and utilization of warehouse. In this study we introduce a three step method to identify goals, integrating them with PW and further using these goals to navigate the warehouse for process analysis and improvements. We further investigate the usability and impact of the method from users' perspective. For that, we have conducted an empirical study based on the evaluation model proposed by Hong et al. [12]. The key benefits that the method offers are: to support goal-based navigation of PW, to retrieve data relevant to the goals and to enhance the ways users analyze and improve business processes.

The rest of the paper is organized as follows: Section 2 briefly reports on work related to ours. In Section 3 the proposed method for process analysis and improvement is introduced. Section 4 presents the evaluation of the proposed approach. Section 7 summarizes the contribution of this study and elicits the directions of future work.

\section{Related Work}

Studies on business process analysis have focused on the utilization of processoriented warehouses by proposing basic toolsets like Business Process Cockpit [8] and architectures like Business Process Intelligence System architecture [13], which allow users to monitor and analyze processes-related data. Other studies on process warehouses have focused on proposing adequate multidimensional data models for this kind of warehouse, such as generic [4], or domain specific (e.g. healthcare, chemical engineering). An analysis of such PW design proposals concluded incompleteness of the presented data models [14]. Also, a recent study [15] contend that these systems don't provide the means for process improvement, therefore improvement can be done solely based on human knowledge and experience.

Business orientation of warehouse: During the last decade a number of efforts have been reported on the business orientation in the data warehouse. At first, Sarda et al. [16] discussed the use of business metadata in data warehouse systems. Following that, Stefanov et. al. [7] extended Sarda's work and introduced an integrated view on data warehouse and enterprise models using "model weaving links". The model weaving approach was also used to make the relationship between data warehouse and enterprise goals visible and accessible [11] through an analysis tool to support and improve data interpretation. However, in these studies, the content scope is restricted to data-orientated information systems and methods that can guide process analysis and improvement are not discussed. 
Methods for process improvement: Recent studies [9, 17, 18] have advocated the need for building methods and frameworks that can guide analysis and improvement of processes. These approaches uses process execution logs or process warehouses as a data source. However, either these approaches have a limited scope (e.g. [18]'s scope is limited to agent assignment), or they do not facilitate extraction and interpretation of the information needed for process analysis and improvement.

This study combines the ideas of the aforementioned related work aiming at making use of process warehouse for goal-driven business process analysis. We propose a well-structured method that differs from existing ones in the way that it integrates goals with process warehouse, while providing the goal structure for utilizing the warehouse to retrieve data for analysis of process performance.

\section{Goal-Driven Process Improvement: The Method}

In this section we introduce our goal-driven process improvement method. The purpose of the method is to facilitate PW designer in integrating goals with PW and process manager to diagnose process related weakness and make changes to the process for possible improvements. The method consists of three steps: build a goal structure (section 3.1), integrate goals into a PW (section 3.2), analyze and improve business process (section 3.3).

\subsection{Step 1: Build Goal Structure}

Our method utilizes the process design framework [19] and the goal decomposition tree (GDT) proposed by [20] in agent programming. The process design framework is used as a reference to ensure that all essential process concepts are addressed and comprehensively represented, as exemplified in [21]. Whereas, GDT is a mechanism that captures both the declarative and procedural aspects of goals, which offers the ability to reason about goals. GDT provides traceability between goals (both top-down and bottom up) using logical inferences through a set of decomposition operators, allowing claims on a goal's achievement based on its sub-goals' achievement. Considering [20] we shift from goal-agent to goal-process coupling. Within the scope of this paper a goal is a state of a process in terms of the quality of a service property that is intended to be achieved (e.g. quality of service: timely, goal: treatment is timely). Figure 1 depicts the workflow of the tasks, followed by their description:

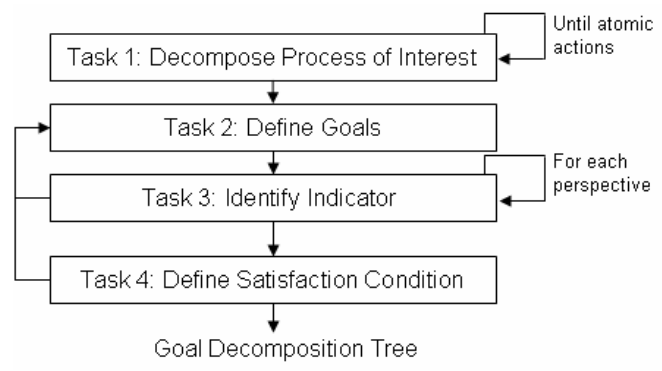

Fig. 1. Workflow of Step 1: Build goal structure 
The first task is to recursively analyze a process from its functional perspective and break it down to atomic actions; where no further process sub-elements can be identified. A Process $\mathrm{P}$ is disaggregated from its functional perspective, $\mathrm{P}=\mathrm{P}_{1} \wedge$ $\mathrm{P}_{2} \wedge \ldots \wedge \mathrm{P}_{\mathrm{n}}$, where $\mathrm{P}_{1}, \mathrm{P}_{2}, \ldots, \mathrm{P}_{\mathrm{n}}$ are sub-process elements of $\mathrm{P}$. For the subsequent subprocess $P_{1}=A_{11} \wedge A_{12} \wedge \ldots{ }^{\wedge} A_{1 n}$, where $A_{11} \ldots A_{1 n}$ are activities of $P_{1}$. Apart from AND decompositions, more operators can be used (e.g. OR, SeqAND, SeqOR etc. [20]).

The second task is to define goals for the process by identifying the quality of service properties needed to be considered for process analysis. Based on the functional decomposition of a process, each goal is expanded into sub-goals, where achievement of the goal relies conventionally on the disaggregated achievement of sub-goals. Let $G$ be the elicited goal of process $P$. The Goal $(P, G)$ is expanded by disaggregation of the process $(\mathrm{P} 1, \mathrm{G} 1)^{\wedge}(\mathrm{P} 2, \mathrm{G} 2)^{\wedge} \ldots{ }^{\wedge}(\mathrm{Pn}, \mathrm{Gn})$.

The third task is to identify criteria for the achievement of goals. It is a bottom-up task in which indicators are set through a cognitive process for each goal by employing the process design framework with four perspectives (functional $\mathrm{F}$, behavioral $\mathrm{B}$, organizational $\mathrm{O}$ and informational $\mathrm{O}$ ). Recall, process design framework covers basic building blocks and ensures a comprehensive representation of process concepts. Therefore, by employing the perspectives we aim for a comprehensive criteria for each goal. For the achievement of goal $\mathrm{G}$ defined on process $\mathrm{P}$, indicators $\mathrm{I} 1, \mathrm{I} 2, \mathrm{I} 3$, I4 are set, relevant to each perspective; $(\mathrm{F}, \mathrm{I} 1),(\mathrm{B}, \mathrm{I} 2)$, $(\mathrm{O}, \mathrm{I} 3),(\mathrm{I}, \mathrm{I} 4)$.

The fourth task is to specify satisfaction conditions (S) for each indicator, where satisfaction conditions of all indicators of a goal form the satisfaction condition of the goal. A satisfaction condition is the desired instance of an indicator that demonstrates goal achievement as defined by stakeholders. Satisfaction condition is a coupling of context and desired value, represented by $\mathrm{S}(\mathrm{C}, \mathrm{V})$. Desired value is the value aimed at being achieved, while context represents the conditions for which the value is valid. For indicators I1, I2, I3, I4 satisfaction conditions S1, S2, S3, S4 are defined respectively. When each satisfaction condition is satisfied, $(\mathrm{I} 1 \mid \mathrm{F} \text { S1) ^ (I2 }=\mathrm{S} 2)^{\wedge}$ $(\mathrm{I} 3 \mid=\mathrm{S} 3)^{\wedge}(\mathrm{I} 4 \mid=\mathrm{S} 4)$ then all satisfaction conditions are logically true, therefore, goal $\mathrm{G}$ is considered to be achieved.

\subsection{Step 2: Integrating Goals with Process Warehouse}

In this step, goals are integrated with a PW to later facilitate the acquisition of goal related information. We propose changes in a PW description at two levels, Schema and Data level.

Schema level: It defines the tables and segments of a table (in terms of attributes) relevant to a goal. This level captures information about the tables (dimension, fact) and attributes that are related with goals, through indicators. Our approach to capture schema level relationships is based on adding a stable structure of tables which captures information about goals, indicators, satisfaction conditions and their relationships with dimension, fact tables. Figure 2 shows the data model for stable structure of tables, which is hardcoded in our prototype (see details in Section 3.3). 


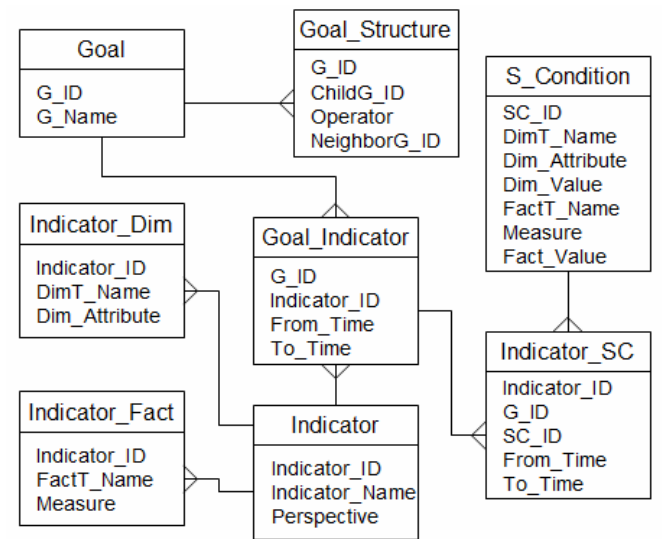

Fig. 2. Data model for stable part of PW

Data level: It defines the instances (in terms of records) of tables (dimension and fact) related to a goal. Our approach to relating records with goals is based on adding bitmaps to the dimension and fact tables that are defined relevant to the goal on the schema level i.e. we negate the addition of bitmaps to each table for each goal to avoid unnecessary increase in size of PW. The bitmap attribute can possess one value, either 1 or 0 representing relevance and non-relevance of a record, respectively. However, the relationship is defined on two levels, Schema and Data, so the bitmap value ( 1 or 0$)$ does not represent the relevance or irrelevance of the complete record. Instead value 1 of a goal's bitmap represents the relevance of the record to the segment of the table defined at schema level.

\section{Step 3: Analyze \& Improve Business Process}

The final step of our method is focused on utilizing PW for process analysis and improvement by identifying weaknesses that hinder goal satisfaction. This step consists of the following tasks: condition identification, goal identification, information analysis, decision elicitation and process change solution. Figure 3 depicts the workflow of the tasks followed by a brief description of each task, due to space limitations.

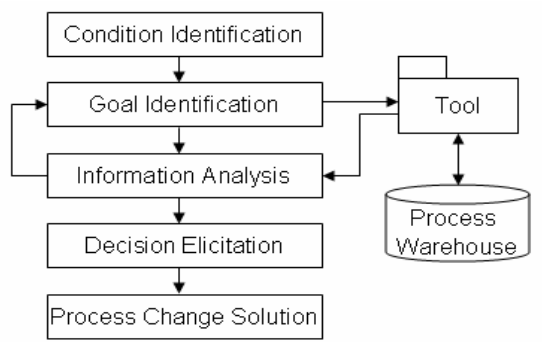

Fig. 3. Workflow for Step 3: Analyze and Improve Business Process 
The first task, condition identification, triggers business process analysis. The reason could be either the occurrence of a process-related event or a predefined temporal state being reached. Consider a simple example, 'registration resource purchases go over budget' as a triggering event for process analysis.

The second task is to select a goal, or a set of goals from the goal structure built in Step 1 and examine their satisfaction against the real process data, stored in the warehouse. Based on the triggering condition, a goal structure is chosen. If the problem concerns only a certain set of sub-processes, or activities, i.e. not the whole process then goal analysis starts from a corresponding node in the goal tree. In case of only a single sub-goal in the tree being considered, all satisfaction conditions for all goal indicators must be satisfied for the (sub)goal to be considered satisfied. In case of more sub-goals being considered, conclusion on their satisfaction depends on the operators used (see Section 3.1. - AND, OR, SeqAND, etc.). In the example, wastage of resource is the main concern on registration sub-process. The chosen goal therefore becomes 'efficient registration', where 'efficiency' is the quality of service property related to wastage of resources.

The third task, information analysis, concerns the analysis of information retrieved from the warehouse. Analyzing the information to reach appropriate conclusions relies on comparing the satisfaction condition with the values (facts) acquired from the processes. If a goal is achieved, further analysis is not required. However, if a goal is not achieved due to values of facts not being equal to satisfaction condition, for each goal in the goal sub-structure the above tasks are repeated until leaf goals are reached. For the chosen goal in the example, the defined tasks need to be repeated for each activity, until the activity where resources are overused is identified.

The fourth task is to elicit the decisions whose realization will result in goal achievement. Input to this manual phase are both goals and the conclusions, while output is a decision taken based on one of the process perspectives. For the example, the decision becomes: reduce the usage of registration equipment in the registration activity, based on informational perspective. It is a cognitive task therefore there is a possibility that more than one decision are reached as a result of this task.

The fifth task is to facilitate the propagation of the obtained decision(s) to the analyzed process a pattern-based approach is used. As shown in the previous section, a decision concerns a change in the process, based on one or more of the process perspectives. As such, a decision gives a rise to a set of atomic process improvement patterns collected and outlined in Table 1.

Table 1. Process improvement patterns

Informational Add resource type, Add resource, Remove resource type, Remove resource, Substitute resource type, Substitute resource, Substitute resource

Organizational Add new responsibility type, Add new responsibility, Remove responsibility type, Remove responsibility, Substitute responsibility type, Substitute responsibility

Functional Add activity/sub-process, Eliminate activity/sub-process, Replace activity/subprocess, Automate activity/ sub-process, Move activity/sub-process

Behavioral Add condition, Remove condition, Substitute condition, Move condition, Add condition, Update condition, Sequence to parallel, Parallel to sequence add concurrency, Reorder sequence, Change Iteration. 
If during process analysis several alternative goals are considered, or several alternative decisions are elicited, it is of benefit to have a mechanism to evaluate the consequences of different process change patterns. One possibility is to evaluate patterns along the four common process impact factors: time, cost, quality, and flexibility identified by Mansar et al. [22]. For an illustration, consider that the goal 'register patient is efficient' is not satisfied due to an overuse of nurses in the patient registration. Before choosing a process improvement pattern, from a set of alternative patterns, it is important to assess the patterns' effect on the process. In Table 2 we have considered two pattern examples: the first, "remove responsibility", and the second, "substitute responsibility type". If a number of nurses are removed from the register patient activity, the time for the registration will increase and since the nurses can be used at some other place the cost of the process will decrease. However, the quality and flexibility will not be affected. To exemplify how different patterns may have different effects, we consider that another option is to substitute the nurses with registrars, who are trained for registration. Now, the activity time will decrease and the quality will increase, because registrars are more skilled than nurses for performing the activity and the activity cost will decrease due to a lower cost of the registrars compared to nurses.

Table 2. Effects of process improvement patterns

\begin{tabular}{lcccc}
\hline Process Improvement Patterns & Time & Cost & Quality & Flexibility \\
\hline Remove responsibility & $+/+$ & $-/-$ & N/A & N/A \\
Substitute responsibility type (nurse by registrar) & $-/-$ & $-/-$ & $+/+$ & N/A \\
\hline
\end{tabular}

+/+ Increase +/- may increase $\quad$-/- Decrease N/A Not applicable

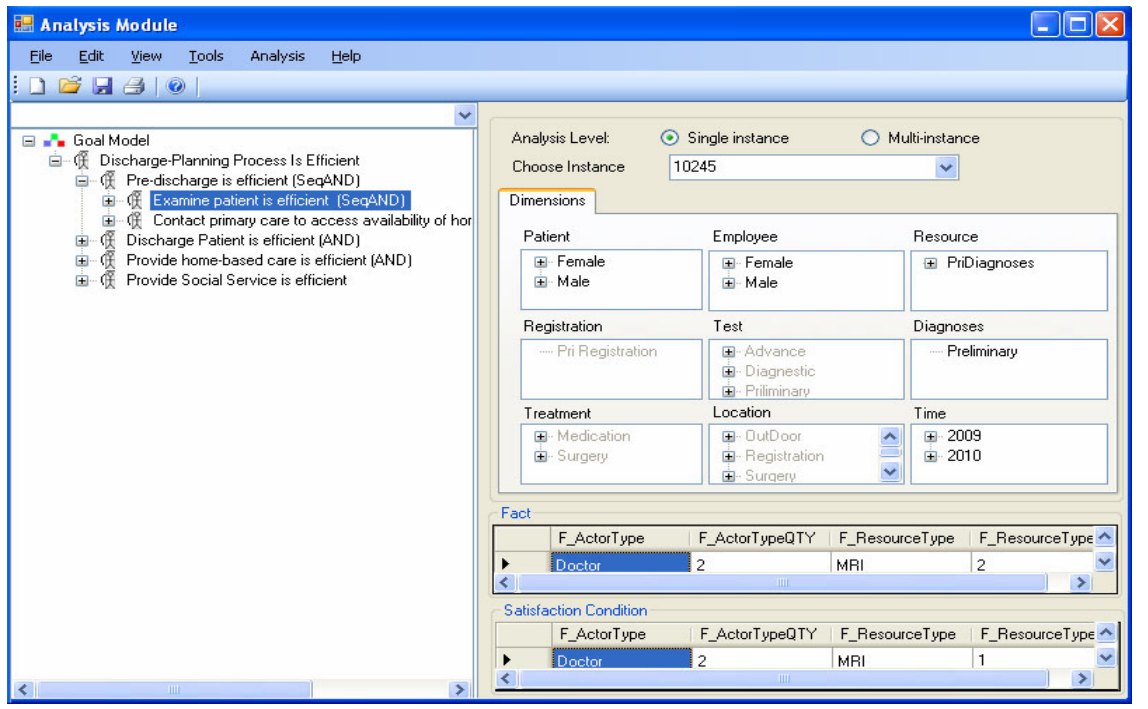

Fig. 4. Prototype of Goal Driven Process Analysis 
To increase the usability of the proposed method, we have developed a prototype, as an instrument to facilitate the addition of goals, integration of goals with PW and goal analysis through a use interface for each task. Due to space limitation a screenshot of implemented prototype is shown in figure 4.

Following the choice of a goal for process analysis, both the corresponding satisfaction condition and the values are extracted from the warehouse. The major benefit of the tool is that it enables users to select goals from the tree (left pane in Figure 4), and set their desired satisfaction conditions for automatic retrieval of data from executed process instances. A user can choose to analyze a specific instance of a process or a set of instances for analysis by using the option given in the left pane.

\section{Method Evaluation}

In this section we briefly describe the empirical study we have conducted to investigate the impact of our proposed method on process analysis. We have adapted the evaluation model developed by Hong, et al. [12], which is based on the Technology Acceptance Model [23], the IS Success Model [24] and factors affecting data warehousing success. For this study, we have considered six out of the eight constructs of Hong et al. model (data quality, accessibility, training, perceived usefulness, perceived ease of use and perceived impact) ${ }^{1}$ as well as all items related to the aforementioned constructs.

\subsection{Research Instrument and Data Collection}

For the empirical study, a PW was implemented for a healthcare process using a proprietary database management system and sample data of 100 patients were used. Based on the goals defined by Swedish Healthcare Institute [25], five goal structures were built, which were then added and linked to the warehouse using the prototype.

Based on an earlier study [26] where one of the authors had participated together with healthcare practitioners in Stockholm, Sweden, we identified eight processrelated problems that trigger the need for process analysis.

Twenty university employees volunteered to participate in the study. A study mentor explained our approach in detail and demonstrated a few examples, followed by a question and answer session to address any individual concern. Participants were then asked to analyze the process for the given problems by using the approach described in section 3.3. Upon completion, participants were asked to fill a post task survey. The survey questionnaire was also adopted from Hong et al. [12] and consisted of 23 construct items (or simply items) as well as an answering scale: strongly disagree (1), simple disagree (2), weak disagree (3), not sure (4), weak agree (5), simple Agree (6) and strongly agree (7).

\footnotetext{
${ }^{1}$ The seventh construct, response time, has not been considered because in our method we don't aim to reduce response time, rather we aim at relevant content retrieval.
} 


\subsection{Data Analysis and Discussion}

The data collected from the empirical study are analyzed to evaluate the use and perceived impact of our method. For analyzing the responses, frequency distribution analysis has been used in order to identify the distribution of responses on a scale of agree, not sure and disagree; where 'agree' encapsulates all those answered strongly agree, simple agree or weak agree and 'disagree' encapsulates all those answered strongly disagree, simple disagree or weak disagree.

Figure 5, shows that our method has overall received positive responses by a large number of participants, which indicates that they perceived our method positively. However, a significant percentage (70\% and 65\%) of participants disagrees with two construct items Q2 and A4. The disagreement with first item indicates that our method does not retrieve irrelevant information i.e. it retrieves relevant information, whereas with second item indicates that the task of retrieving the information is not effortless.

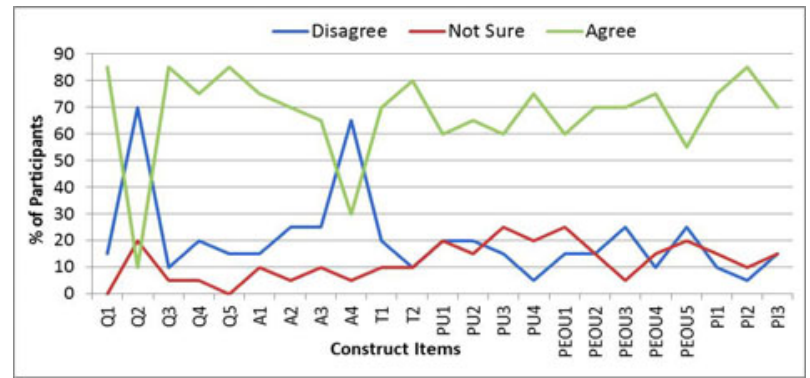

Fig. 5. Frequency distribution of construct items

Figure 6 summarizes the results of users' perception of the method in terms of the six constructs. Values of each construct are calculated by taking a mean of the items belonging to the construct. More than $75 \%$ of the participants agree that the perceived impact is positive.

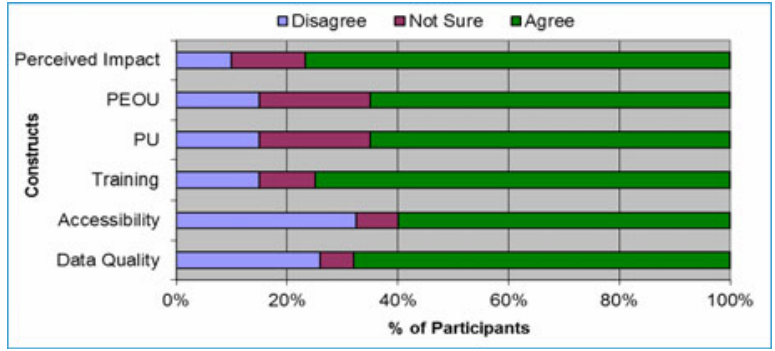

Fig. 6. Frequency distribution of constructs

To evaluate whether the participants' background knowledge influences their perception of the method or not, we have classified participants into two groups; experienced and novice users. Users having passed a course on data warehousing and 
having participated in a data warehousing project belong to the experienced group while users without any background knowledge of data warehousing belong to the novice group. Information about participants' background knowledge was collected during the study. Our sample includes 8 experienced and 12 novice users.

Figure 7 shows the difference between experienced and novice users who agree on the construct items. According to the figure, experienced users agreed with the construct items in a larger percentage than novice ones, indicating that that these construct items were better perceived by experienced users than novice users.

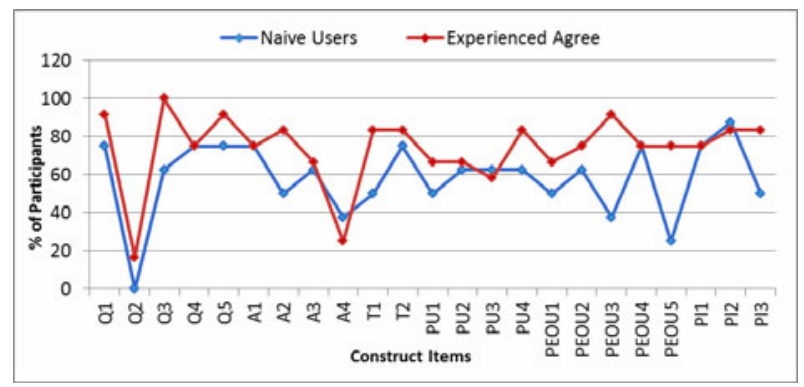

Fig. 7. Comparison of user groups on construct items

Figure 8 summarizes the results of users' perception of the method when considering the six constructs. The results show a greater difference in the perception of data quality, training and perceived ease of use than in accessibility, perceived usefulness and perceived impact.

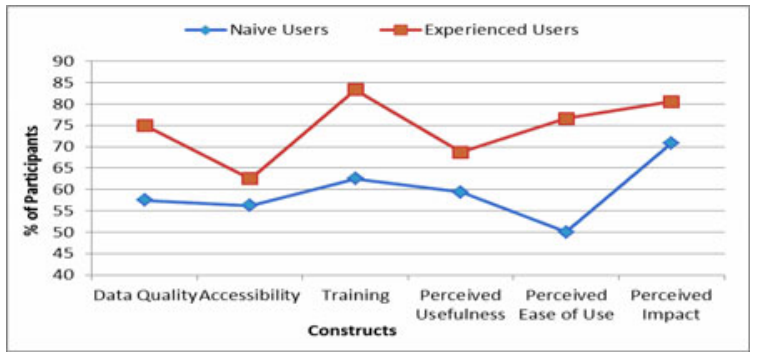

Fig. 8. Comparison of user groups on constructs

\section{Conclusion}

In this paper, we have proposed a three step method for goal-driven analysis of business process models aiming at eliciting possible improvement directions. In the first step a goal structure is defined for the process of interest. In the second step goals are integrated with process warehouse and in the third step goal structures are used for process analysis and improvement. To evaluate users' perceptions of our method's impact on process analysis, we have conducted an empirical study based upon an existing evaluation model. 
Based on the results of the study we can conclude, a) the perceived impact of our method is positive; b) the construct items are better perceived by experienced users than novice users, c) experienced users perceived data quality, training and perceived ease of use more than accessibility, perceived usefulness and perceived impact.

Regarding future work, we are aiming for a refinement and further evaluation of the method. In addition, we intend to consider the augmentation of the automation for the proposed method steps.

\section{References}

[1] Castellanos, M., Simitsis, A., Wilkinson, K., Dayal, U.: Automating the Loading of Business Process Data Warehouse. In: Proceedings of the 12th ACM International Conference on Extending Database Technology (EDBT 2009), Russia, pp. 612-623 (2009)

[2] Mansmann, S., Neumuth, T., Scholl, M.H.: Multidimensional data modeling for business process analysis. In: Parent, C., Schewe, K.-D., Storey, V.C., Thalheim, B. (eds.) ER 2007. LNCS, vol. 4801, pp. 23-38. Springer, Heidelberg (2007)

[3] List, B., Schiefer, J., Tjoa, A.M., Quirchmayr, G.: The Process Warehouse: A Data Warehouse Approach for BPM. In: Abramowicz, W., Zurada, J. (eds.) Selected Aspects of Knowledge Discovery for Business Information Systems. Kluwer Academic, USA (2000)

[4] Casati, F., Castellanos, M., Dayal, U., Salazar, N.: A Generic Solution for Warehousing Business Process Data. In: Proceedings of the 33rd VLDB 2007, Austria, pp. 1128-1137 (2007)

[5] Shahzad, K., Zdravkovic, J.: A goal-oriented approach for business process improvement using process warehouse data. In: Persson, A., Stirna, J. (eds.) PoEM 2009. Lecture Notes in Business Information Processing, vol. 39, pp. 84-98. Springer, Heidelberg (2009)

[6] Muehlen, M., Shapiro, R.: Business Process Analytics. In: Brocke, J., Rosemann, M. (eds.) Handbook on Business Process Management 2: Strategic Alignment, Governance, People and Culture (International Handbooks on Information Systems). Springer, Heidelberg (2010)

[7] Stefanov, V., List, B.: Explaining Data Warehouse Data to Business Users - A Model Based Approach to Business Metadata. In: Proceedings of the ECIS 2007, Switzerland, pp. 2062-2073 (2007)

[8] Sayal, M., Casati, F., Dayal, U., Shan, M.C.: Business Process Cockpit. In: Proceedings of the 28th International Conf. on Very Large Databases (VLDB 2002), Hong Kong (2002)

[9] Pourshahid, A., Amyot, D., Peyton, L., et al.: Business process management with the user requirements notation. Electronic Commerce Research 9(4), 269-316 (2009)

[10] Shahzad, K., Zdravkovic, J.: Towards Goal-driven access to Process Warehouse: Integrating Goals with Process Warehouse for Business Process Analysis. To appear in the Proceedings of the Fifth IEEE International Conference on Research Challenges in Information Sciences (RCIS), Guadeloupe, France (2011)

[11] Stefanov, V., Beate List, B.: Business Metadata for the Data Warehouse - Weaving Enterprise Goals and Multidimensional Models. In: Proceedings of the International Workshop on Models for Enterprise Computing (IWMEC) at the EDOC 2006, Hong Kong (2006) 
[12] Hong, S., Katerattanakul, P., Hong, S., Cao, Q.: Usage and Perceived Impact of Data Warehouses: A Study of Korean Financial Companies. International Journal of Information Technology and Decisin Making 5(2), 297-315 (2006)

[13] An, L., Yan, J., Tong, L.: Business Process Intelligence System: Architecture and Data Models. In: Proc. of the 6th Wuhan International Conference on e-business, China (2007)

[14] Shahzad, K., Johannesson, P.: An Evaluation of Process Warehousing Approaches for Business Process Analysis. In: Proceedings of the 5th ACM-EOMAS in conjunction with CAiSE 2009. CEUR-WS Proceedings, vol. 458, pp. 1-14. ACM Press, New York

[15] Pourshahid, A., Mussbacher, G., Amyot, D., Weiss, M.: Toward an aspect-oriented framework for business process improvement. International Jouranal of Electronic Business 8(3), 233-259 (2010)

[16] Sarda, N.L.: Structuring Business Metadata in Data Warehouse Systems for Effective Business Support, http://arxiv.org/abs/cs/0110020 (last access on December 19, 2010)

[17] Shahzad, K., Zdravkovic, J.: A Decision-model Based Approach to Business Process Improvement. In: Proceedings of the IEEE-SMC 2010, Istanbul, Turkey, pp. 810-818 (2010)

[18] Jablonski, S., Talib, R.: Agent assignment for process management: Pattern based agent performance evaluation. In: Cao, L., Gorodetsky, V., Liu, J., Weiss, G., Yu, P.S. (eds.) ADMI 2009. LNCS, vol. 5680, pp. 155-169. Springer, Heidelberg (2009)

[19] Curtis, B., Kellner, M., Over, J.: Process Modeling. Comm. of ACM 35(9), 75-90 (1992)

[20] Simon, G., Mermet, B., Fournier, D.: Goal decomposition tree: An agent model to generate a validated agent behaviour. In: Baldoni, M., Endriss, U., Omicini, A., Torroni, P. (eds.) DALT 2005. LNCS (LNAI), vol. 3904, pp. 124-140. Springer, Heidelberg (2006)

[21] List, B., Korherr, B.: An Evaluation of Conceptual Business Process Modelling languages. In: Proceedings of the ACM Symposium on Applied Computing (SAC 2006), NY, USA, pp. 1532-1539 (2006)

[22] Mansar, S.L., Reijers, H.A.: Best Practices in business process redesign: use and impact. Business Process Management Journal 13(2), 193-213 (2007)

[23] Davis, D.F., Bagozzi, R.P., Warshaw, P.R.: User acceptance of Computer Technology: A Comparison of two Theoretical Models. Management Science 35(8), 982-1003 (1989)

[24] De Lone, W.H., McLean, E.R.: Information Systems Success: The Quest for the Dependent variable. Information Systems Research 3(1), 60-95 (1992)

[25] Institute of Medicine. Crossing the Quality Chasm: A New Health System for the 21st Century, http://www.iom.edu/CMS/8089/5432.aspx (last accessed on July 30, 2010)

[26] Hägglund, M., Henkel, M., Zdravkovic, J., et al.: A New Approach for Goal-oriented Analysis of Healthcare Processes. Stud Health Tech. Inform. 160(2), 1225-1251 (2010) 